\title{
A new analytical method for solving the problem of one-dimensional transient heat conduction in a slab by using the temperature solutions of semi-infinite solids
}

\author{
Sumio KATO* and Shoichi MATSUDA* \\ *Faculty of Engineering, University of the Ryukyus \\ 1 Senbaru, Nishihara, Okinawa 903-0213, Japan \\ E-mail: katosumi@tec.u-ryukyu.ac.jp
}

Received: 15 February 2017; Revised: 24 April 2017; Accepted: 29 May 2017

\begin{abstract}
A new simple analytical method for solving the problem of one-dimensional transient heat conduction in a slab of finite thickness is proposed, in which the initial temperature is assumed zero or constant and the boundary surfaces are assumed to be at constant temperature, constant heat flux, or insulated. In this method, the solution is expressed by an infinite series representation, each term of which is the temperature solution of the corresponding initial-boundary value problem for the semi-infinite solid. Each semi-infinite solid extends to infinity in the positive or negative direction of the $x$ axis and the surface is located at various positions along the $x$ axis. Each term and the partial sum in the infinite series automatically satisfy the heat conduction equation and the initial condition. The solution is easily constructed so that the boundary values of the partial sum converge to those of the heat conduction problem as the number of terms $N$ increases to infinity. The basic concept of the solution method for the problem of one-dimensional transient heat conduction in a slab is described. The solution method is applied to various initial-boundary value problems. The formulas of the typical solutions by this method are found to be the same as those of the solutions obtained by other literature using the method of Laplace transformation, which supports the validity of the new solution method proposed in this paper. The usefulness of this method is also examined.
\end{abstract}

Key words : Heat conduction, Analytical method, One-dimensional transient heat conduction, Initial-boundary value problem, Semi-infinite solid, Slab

\section{Introduction}

Heat transfer by conduction (steady state or transient conduction) is of great importance in heat transfer engineering. As for the transient conduction, one-dimensional heat conduction problems with initial and boundary conditions are fundamental.

Various methods may be used to find numerical and analytical solutions for one-dimensional transient heat conduction problems. Numerical methods (Chapra and Canale, 2010) (Farlow, 1993) (Lewis et al., 2004) include the finite difference method, the finite element method, etc. Analytical methods (Brown and Chuchill, 1993) (Constanda, 2002) (Churchill, 1958) (Farlow, 1993) include the method of separation of variables, the Laplace transformation, the method of eigenfunction expansion, the method of Green's function, etc.

Numerical and analytical solutions have both advantages and disadvantages (Farlow, 1993). Numerical methods give the solutions for complicated problems with nonlinearity for which analytical solutions are not known. However, the amount of information from numerical solutions is rather limited or small compared with that of analytical solutions. As for analytical solutions, analytical solutions tell us how physical parameters, initial and boundary conditions affect the solutions with little effort compared with the case of numerical solutions. Analytical solutions are accurate and can be used to verify computer solutions obtained by numerical methods using newly developed programs. However, 
problems to which analytical methods can be applicable are limited to rather simple ones, and mathematical methods for analytical solutions are relatively complicated and difficult.

In this paper, we propose a new simple analytical method for solving the problem of one-dimensional transient heat conduction with initial and boundary conditions for a slab of finite thickness. Thermo-physical properties of the material are assumed constant. The initial temperature $T_{\mathrm{i}}$ is assumed zero or constant. (When $T_{\mathrm{i}}$ is not zero, the problem is transformed to the case of $T_{\mathrm{i}}=0$.) The boundary surfaces are assumed to be at constant temperature, constant heat flux, or insulated. In this method, the solution is expressed by an infinite series representation, where each term is the temperature solution (temperature function) of the corresponding initial-boundary value problem for the semi-infinite solid. The semi-infinite solid for each term extends to infinity in the positive or negative direction of the $x$ axis and the surface is located at various positions along the $x$ axis.

The solution by the infinite series representation is constructed so that the boundary values of the partial sum of $N$ terms, which satisfies the heat conduction equation and the initial condition, converge to the values of the boundary conditions as $N$ increases to infinity. The construction of the solution is easy to conduct if we draw the graphic curves of the temperature functions for several terms and the sum of them.

As far as the authors know, this solution method has not been proposed before yet. In the following, the basic concept of the solution method for one-dimensional transient heat conduction problems is described. The solution method is applied to various initial-boundary value problems. The formulas of the typical solutions by this method are found to be the same as those of the solutions obtained by other literature using the method of Laplace transformation, which supports the validity of the new solution method proposed in this paper. The usefulness of this method in the field of mechanical engineering is also examined.

\section{Nomenclature}

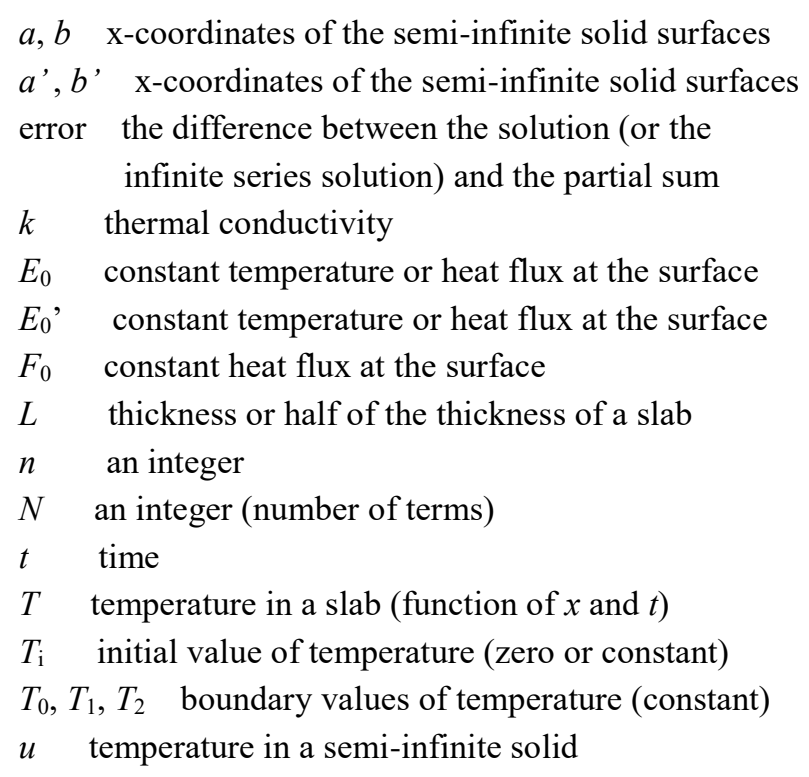

(function of $x$ and $t$ )

$u_{i} \quad i$ th term of the infinite series for the solution

$U_{\mathrm{L}}, U_{\mathrm{R}}$ temperature function (temperature solution) in a semi-infinite solid which extends to the negative and positive directions of the $x$ axis, respectively

$x \quad x$ coordinate

Greek letters

$\alpha$ thermal diffusivity

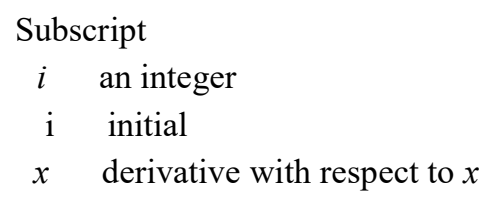

\section{The basic concept of the solution method}

The basic concept of the solution method for one-dimensional transient heat conduction problems in a slab is explained. The procedure for the method consists of the following three steps: (1) Definition of the heat conduction problem in a slab, (2) Definition of the temperature functions in semi-infinite solids, and (3) Construction of the infinite series for the solution. The typical procedure is shown below.

\subsection{Definition of the heat conduction problem in a slab}

A slab with finite thickness, in which the initial temperature $T_{\mathrm{i}}$ is zero or constant, is considered. It is assumed that the boundary surfaces are at constant temperature, constant heat flux, or insulated. It is also assumed that the thermo-physical properties of the slab are constant. By considering these conditions, the initial-boundary value problem of heat conduction in a slab is defined. 


\subsection{Definition of the temperature functions in semi-infinite solids}

The temperature function is the temperature solution in a semi-infinite solid, which is a candidate function for use in the infinite series solution. We consider the temperature function (temperature solution) in a semi-infinite solid defined in $a \leq x<\infty$, where the surface is located at $x=a$. The initial and boundary value conditions for the semi-infinite solid are based on those of the corresponding slab. The initial temperature $T_{\mathrm{i}}$ for the semi-infinite solid is zero or constant. (When $T_{\mathrm{i}}$ is not zero, the problem is transformed to the case of $T_{\mathrm{i}}=0$; See Sec. 3.5.) The boundary surface of the semi-infinite solid is at constant temperature or constant heat flux, which is determined based on the boundary conditions of the corresponding slab. With various values of $a$, we have the temperature functions expressed by $U_{\mathrm{R}}\left(E_{0}, a, x, t\right)$, where $E_{0}$ corresponds to the constant temperature or constant heat flux at the surface. In the similar way, we define $U_{\mathrm{L}}\left(E_{0}, b, x, t\right)$ in $-\infty<x \leq b$, where the surface is located at $x=b$ with various values of $b$. $U_{\mathrm{R}}\left(-E_{0}\right.$, $a, x, t)$ and $U_{\mathrm{L}}\left(-E_{0}, b, x, t\right)$ are also the candidate temperature functions.

In some problems, $U_{\mathrm{R}}\left( \pm E_{0}, a^{\prime}, x, t\right)$ and $U_{\mathrm{L}}\left( \pm E_{0}, b^{\prime}, x, t\right)$ are used in addition to $U_{\mathrm{R}}\left( \pm E_{0}, a, x, t\right)$ and $U_{\mathrm{L}}\left( \pm E_{0}, b, x\right.$, $t$ ). A typical example of this case is shown in Sections 3.3.

\subsection{Construction of the infinite series for the solution}

The solution $T(x, t)$ is assumed to be expressed by an infinite series representation as shown below, each term of which is the temperature function (temperature solution) for a semi-infinite solid

$$
T(x, t)=\sum_{i=1}^{\infty} u_{i}(x, t)
$$

where $u_{i}(x, t)$ is selected from the candidate temperature functions of $U_{\mathrm{R}}\left( \pm E_{0}, a, x, t\right)$ and $U_{\mathrm{L}}\left( \pm E_{0}, b, x, t\right)$, with various values of $a$ and $b$. In some problems, $U_{\mathrm{R}}\left( \pm E_{0}, a^{\prime}, x, t\right)$ and $U_{\mathrm{L}}\left( \pm E_{0}, b^{\prime}, x, t\right)$ are also the temperature functions. Each term and the partial sum of $N$ terms satisfy the heat conduction equation and the initial condition. The infinite series is constructed so that the boundary values of the partial sum of $N$ terms converge to the values of the boundary conditions as $N$ increases to infinity. Drawing graphic curves of the temperature functions and the sum helps to construct the series easily. Concept of the infinite series solution consisting of the temperature function (temperature solution) in each semi-infinite solid is shown in Fig. 1.

In obtaining the solutions for the problems in this paper, it is assumed that the solution by the infinite series representation obtained with this method converges and satisfies the corresponding initial-boundary value problem. The validity of the solution method is examined in Chapter 4.

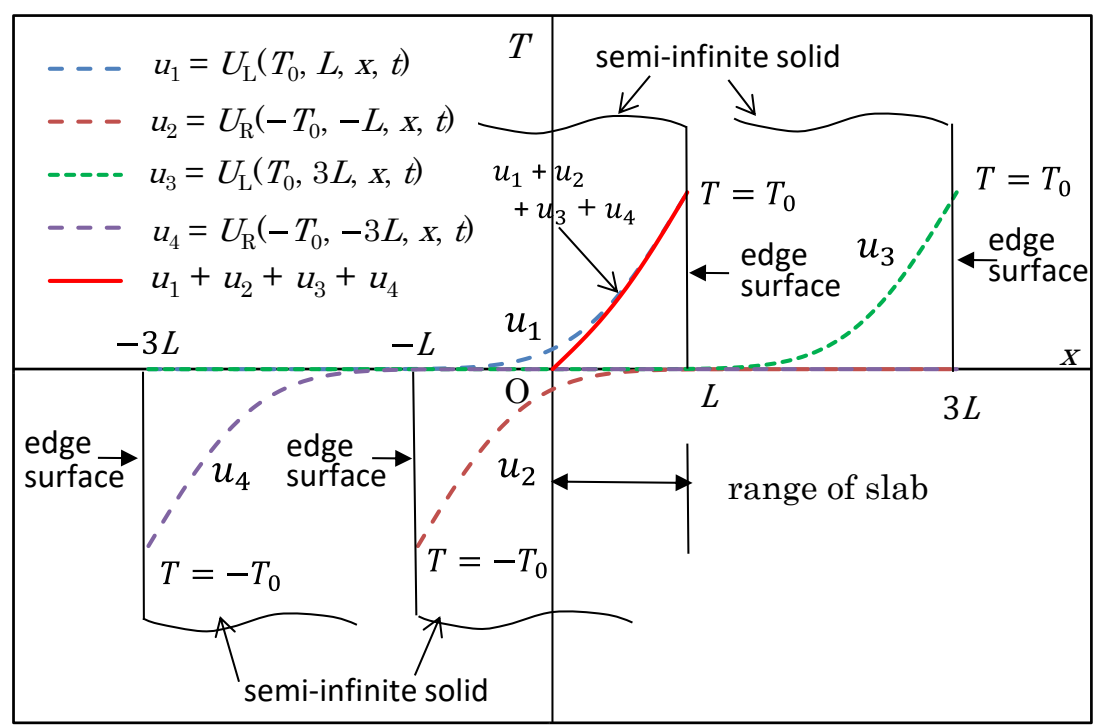

Fig. 1 Concept of the infinite series solution consisting of the temperature functions (temperature solutions) in semi-infinite solids 


\section{Application of the solution method to various problems}

In this chapter, applications of the solution method to various one-dimensional heat conduction problems of a slab are presented. The initial temperature $T_{\mathrm{i}}$ is assumed zero or constant. (When $T_{\mathrm{i}}$ is not zero, see Sec. 3.5.) The boundary surfaces are assumed to be at constant temperature, constant heat flux, or insulated.

\subsection{Example 1 (A slab with zero initial temperature and edge surfaces kept at zero and constant temperatures)}

1) Definition of the heat conduction problem in a slab

Consider a slab $0 \leq x \leq L$ with zero initial temperature, and with the surfaces $x=0$ and $x=L$ kept at zero temperature and constant temperature $T_{0}$, respectively. The temperature in the slab is the solution of the following initial-boundary value problem.

$$
\begin{array}{lc}
\frac{\partial T}{\partial t}=\alpha \frac{\partial^{2} T}{\partial x^{2}} & (0<x<L, t>0) \\
T(x, 0)=0 & (0<x<L) \\
T(0, t)=0, & T(L, t)=T_{0} \quad(t>0)
\end{array}
$$

2) Definition of the temperature functions in semi-infinite solids

We consider the temperature distribution in a semi-infinite solid defined in $a \leq x<\infty$ with zero initial temperature. The surface is located at $x=a$ and kept at a constant temperature $T_{0}$. The initial-boundary value problem for the temperature function $u(x, t)=U_{\mathrm{R}}\left(T_{0}, a, x, t\right)$ is

$$
\begin{array}{cc}
\frac{\partial u}{\partial t}=\alpha \frac{\partial^{2} u}{\partial x^{2}} & (x>a, t>0) \\
u(x, 0)=0 & (x>a) \\
u(a, t)=T_{0} & (t>0) \\
u(x, t) \rightarrow 0 \text { as } x \rightarrow \infty & (t>0)
\end{array}
$$

(Eqs. (3.1-2)a - (3.1-2)d for $a=0$ are given by Churchill (1958).) The solution for the problem is

$$
u(x, t)=U_{R}\left(T_{0}, a, x, t\right)=T_{0} \operatorname{erfc}\left(\frac{x-a}{2 \sqrt{\alpha t}}\right)
$$

(Eq. (3.1-3) for $a=0$ is given by Carslaw and Jaeger (1959), and Churchill (1958).) In the similar way, the temperature function $U_{\mathrm{L}}\left(T_{0}, b, x, t\right)$ is defined to be the solution of the following initial-boundary value problem, where the solid is defined in $-\infty<x \leq b$

The solution for the problem is

$$
\begin{array}{cc}
\frac{\partial u}{\partial t}=\alpha \frac{\partial^{2} u}{\partial x^{2}} & (x<b, t>0) \\
u(x, 0)=0 & (x<b) \\
u(b, t)=T_{0} & (t>0) \\
u(x, t) \rightarrow 0 \text { as } x \rightarrow-\infty \quad(t>0)
\end{array}
$$

$$
u(x, t)=U_{L}\left(T_{0}, b, x, t\right)=T_{0} \operatorname{erfc}\left(\frac{b-x}{2 \sqrt{\alpha t}}\right)
$$

In addition, $U_{\mathrm{R}}\left(-T_{0}, a, x, t\right)\left(=-U_{\mathrm{R}}\left(T_{0}, a, x, t\right)\right)$ and $U_{\mathrm{L}}\left(-T_{0}, a, x, t\right)\left(=-U_{\mathrm{L}}\left(T_{0}, b, x, t\right)\right)$ are also defined as the temperature functions for the construction of the solution.

The temperature functions have the following characteristics [double signs in same order for (1) - (5)]: (1) $U_{\mathrm{R}}\left( \pm T_{0}\right.$, $a, x, t)$ is symmetric with $U_{\mathrm{L}}\left( \pm T_{0}, b, x, t\right)$ with respect to the line of $x=(a+b) / 2$. (2) $U_{\mathrm{R}}\left( \pm T_{0}, a, x, t\right)$ is symmetric with $U_{\mathrm{L}}\left(\mp T_{0}, b, x, t\right)$ with respect to the point $(x=(a+b) / 2, T=0)$, and $U_{\mathrm{R}}\left( \pm T_{0}, a, x, t\right)+U_{\mathrm{L}}\left(\mp T_{0}, b, x, t\right)=0$ for $x=(a+$ 
b)/2 with $a \leq b$. (3) $U_{\mathrm{R}}\left( \pm T_{0}, a, x, t\right)$ is symmetric with $U_{\mathrm{L}}\left(\mp T_{0},-a, x, t\right)$ with respect to the point $(x=0, T=0)$. (4) $U_{\mathrm{R}}\left( \pm T_{0},-a, x, t\right)+U_{\mathrm{L}}\left(\mp T_{0}, a, x, t\right)=0$ for $x=0$ with $a \geq 0$. (5) For constant values of $T_{0}$ and $t$, any of $U_{\mathrm{R}}\left( \pm T_{0}, a, x\right.$, $t)$ and $U_{\mathrm{L}}\left( \pm T_{0}, b, x, t\right)$ with various values of $a$ and $b$ coincides to each other by parallel movement along the $x$ axis, horizontal or vertical inversion, or combination of these. This means that the temperature functions with various values of $a$ and $b$ have the same shape for the same $T_{0}$ and $t$.

3) Construction of the infinite series for the solution

The solution $T(x, t)$ for the initial-boundary value problem, Eqs. (3.1-1)a - (3.1-1)c, is assumed to be expressed by

$$
T(x, t)=\sum_{i=1}^{\infty} u_{i}(x, t)
$$

where $u_{i}$ is selected from the candidate temperature functions of $U_{\mathrm{R}}\left( \pm T_{0}, a, x, t\right)$ and $U_{\mathrm{L}}\left( \pm T_{0}, b, x, t\right)$ with various values of $a$ and $b$. It is apparent that $u_{i}$ satisfies the heat conduction equation of Eq. (3.1-1)a and the initial condition of Eq. (3.1-1)b. Then the partial sum of $N$ terms also satisfies the same Eqs (3.1-1)a and (3.1-1)b. The temperature function and the value of $a$ or $b$ for each $u_{i}$ are determined so that the differences at both surfaces of the slab between the partial sum of $N$ terms and the boundary values converge to zero as $N$ increases to infinity. It follows that the infinite series would satisfy the initial-boundary value problem of Eqs. (3.1-1)a - (3.1-1)c.

Based on the above consideration, the first term $u_{1}$ is determined so that the value of $u_{1}$ is equal to $T_{0}$ at $x=L$. Then $u_{1}$ is set as

$$
u_{1}=U_{L}\left(T_{0}, L, x, t\right)=T_{0} \operatorname{erfc}\left(\frac{L-x}{2 \sqrt{\alpha t}}\right)
$$

The errors of $u_{1}$ at the boundary surfaces of $x=0$ and $x=L$ are $T_{0} \operatorname{erfc}(L /(2 \sqrt{\alpha t}))$ and 0 , respectively. The maximum absolute error at the boundary surfaces is $T_{0} \operatorname{erfc}(L /(2 \sqrt{\alpha t})) \cdot u_{1}$ is shown schematically in Fig. 2. (The error is defined to be the difference between the solution (or the infinite series solution) and the partial sum.)

The second term $u_{2}$ is determined so that the value of $u_{1}+u_{2}$ at $x=0$ becomes zero. Then $u_{2}$ is set as

$$
u_{2}=U_{R}\left(-T_{0},-L, x, t\right)=-U_{R}\left(T_{0},-L, x, t\right)=-T_{0} \operatorname{erfc}\left(\frac{L+x}{2 \sqrt{\alpha t}}\right)
$$

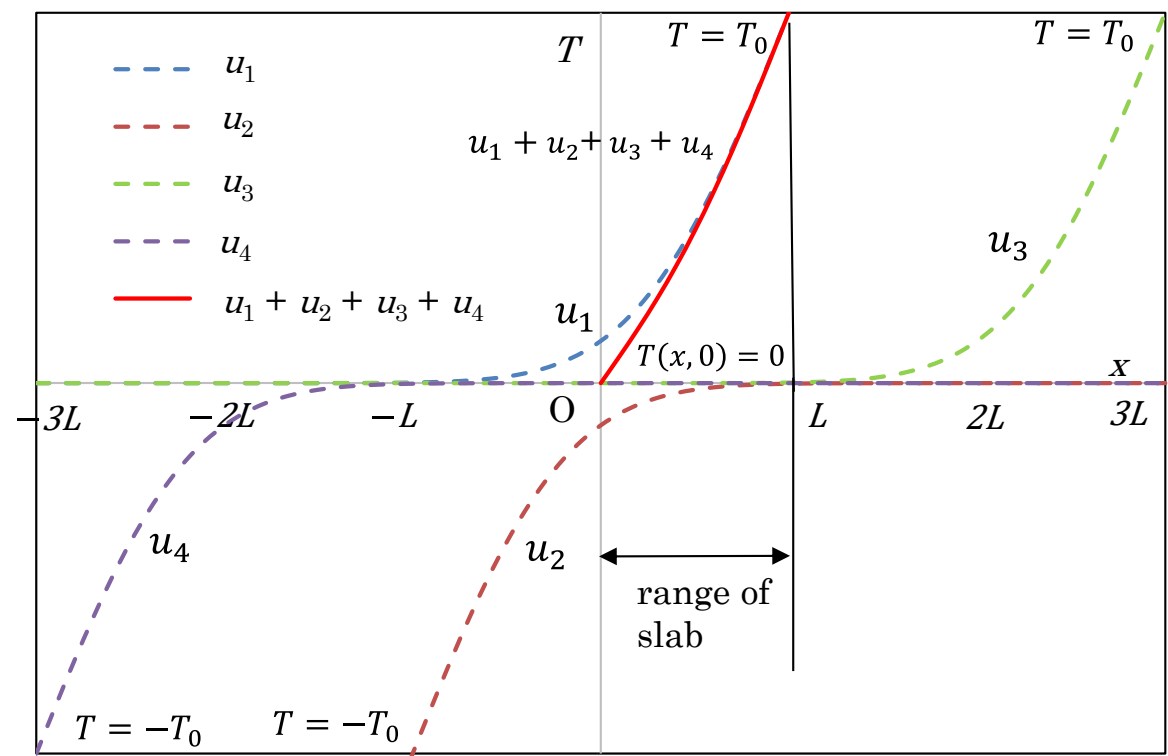

Fig. $2 u_{1}, u_{2}, u_{3}, u_{4}$ and $u_{1}+u_{2}+u_{3}+u_{4}$ (Example 1)

The errors of $u_{1}+u_{2}$ at the boundary surfaces of $x=0$ and $x=L$ are 0 and $-T_{0} \operatorname{erfc}(2 L /(2 \sqrt{\alpha t}))$, respectively. The maximum absolute error at the boundary surfaces of $x=0$ and $x=L$ for $u_{1}+u_{2}$, is $T_{0} \operatorname{erfc}(2 L /(2 \sqrt{\alpha t}))$, which is less 
than that for $u_{1}, T_{0} \operatorname{erfc}(L /(2 \sqrt{\alpha t})) . u_{2}$ is symmetric with $u_{1}$ with respect to the point $\mathrm{O}(x=0, T=0)$. $u_{2}$ is shown schematically in Fig. 2.

The third term $u_{3}$ is determined so that the value of $u_{1}+u_{2}+u_{3}$ at $x=L$ becomes $T_{0} .\left(u_{2}+u_{3}\right.$ becomes zero at $x=L$. $)$ Then $u_{3}$ is set as

$$
u_{3}=U_{L}\left(T_{0}, 3 L, x, t\right)=T_{0} \operatorname{erfc}\left(\frac{3 L-x}{2 \sqrt{\alpha t}}\right)
$$

The errors of $u_{1}+u_{2}+u_{3}$ at the edge surfaces of $x=0$ and $x=L$ are $T_{0} \operatorname{erfc}(3 L /(2 \sqrt{\alpha t}))$ and 0 , respectively. It is seen that the maximum absolute error at the boundary surfaces of $x=0$ and $x=L$ for $u_{1}+u_{2}+u_{3}$, is $T_{0} \operatorname{erfc}(3 L /(2 \sqrt{\alpha t}))$, which is less than that for $u_{1}+u_{2}, T_{0} \operatorname{erfc}(2 L /(2 \sqrt{\alpha t})) . u_{3}$ is shown schematically in Fig. 2 .

In the same way, other terms can be determined. The maximum absolute error for the partial sum of $N$ terms is $T_{0} \operatorname{erfc}(N L /(2 \sqrt{\alpha t}))$, which decreases monotonically and converges to zero as $N \rightarrow \infty$. $u_{4}\left(=-T_{0} \operatorname{erfc}[(3 L+x) /\right.$ $(2 \sqrt{\alpha t})])$ and $u_{1}+u_{2}+u_{3}+u_{4}$ are shown in Fig. 2. Then the formula of the solution for Eqs. (3.1-1)a - (3.1-1)c becomes

$$
T(x, t)=T_{0} \sum_{n=0}^{\infty}\left(\operatorname{erfc} \frac{(2 n+1) L-x}{2 \sqrt{\alpha t}}-\operatorname{erfc} \frac{(2 n+1) L+x}{2 \sqrt{\alpha t}}\right)
$$

This formula for the solution is the same as that of the same problem (Carslaw and Jaeger, 1959), which was obtained by the method of Laplace transformation. The solution in the case of $\alpha=1$ and $L=1$ is also given by Churchill (1958) using the method of Laplace transformation, which is the same as Eq. (3.1-10) with $\alpha=1$ and $L=1$.

\subsection{Example 2 (A slab with zero initial temperature and edge surfaces kept at constant and zero temperatures)}

Consider a slab with zero initial temperature, and with the surfaces $x=0$ and $x=L$ kept at a constant value of $T_{0}$ and zero temperature, respectively. The initial-boundary value problem is

$$
\begin{aligned}
& \frac{\partial T}{\partial t}=\alpha \frac{\partial^{2} T}{\partial x^{2}} \quad(0<x<L, t>0) \\
& T(x, 0)=0 \quad(0<x<L) \\
& T(0, t)=T_{0}, \quad T(L, t)=0 \quad(t>0)
\end{aligned}
$$

Eqs. (3.2-1)a and (3.2-1)b are the same as Eqs. (3.1-1)a and (3.1-1)b in Example 1, respectively. The boundary conditions of Eq. (3.2-1)c are the inverse of those of Eq. (3.1-1)c in Example 1.

The solution for Eqs. (3.2-1)a - (3.2-1)c can be obtained in the similar way to Example 1. Then

$$
T(x, t)=T_{0} \sum_{n=0}^{\infty}\left(\operatorname{erfc} \frac{2 n L+x}{2 \sqrt{\alpha t}}-\operatorname{erfc} \frac{2(n+1) L-x}{2 \sqrt{\alpha t}}\right)
$$

Because the solution of Example 2 is symmetric to that of Example 1 with respect to the line of $x=L / 2$, we can also obtain the same solution easily by replacing $x$ by $L-x$ in Eq. (3.1-10), yielding Eq. (3.2-2).

\subsection{Example 3 (A slab with zero initial temperature and two edge surfaces kept at constant temperatures of $T_{1}$ and $T_{2}$, respectively)}

Two kinds of methods (Method1 and Method2) for the solution are described. In Method1, each term of the infinite series solution is determined one by one in the similar way to that of Example 1. In Method2, the solution is obtained directly from the results of Examples 1 and 2.

\subsubsection{Method1}

1) Definition of the heat conduction problem in a slab

Consider the problem of heat flow in a slab $-L \leq x \leq L$ with edge surfaces of $x=-L$ and $x=L$ kept at constant temperatures of $T_{1}$ and $T_{2}$, respectively, when the initial temperature is zero throughout. Then the initial-boundary value problem is 


$$
\begin{gathered}
\frac{\partial T}{\partial t}=\alpha \frac{\partial^{2} T}{\partial x^{2}} \\
T(x, 0)=0 \quad(-L<x<L, t>0) \\
T(-L, t)=T_{1}, \quad T(L, t)=T_{2} \quad(t>0)
\end{gathered}
$$

2) Definition of the temperature functions in semi-infinite solids

The temperature functions for constructing the solution of Eqs. (3.3-1)a - (3.3-1)c are of the same type as those in Example 1 (Sec. 3.1). The temperature functions in Example 3 are

$$
\begin{aligned}
& U_{\mathrm{R}}\left( \pm T_{1}, a, x, t\right)= \pm T_{1} \operatorname{erfc}\left(\frac{x-a}{2 \sqrt{\alpha t}}\right) \\
& U_{\mathrm{L}}\left( \pm T_{1}, b, x, t\right)= \pm T_{1} \operatorname{erfc}\left(\frac{b-x}{2 \sqrt{\alpha t}}\right)
\end{aligned}
$$

In addition, $U_{\mathrm{R}}\left( \pm T_{2}, a^{\prime}, x, t\right)$ and $U_{\mathrm{L}}\left( \pm T_{2}, b^{\prime}, x, t\right)$ are also set as the temperature functions for the construction of the solution.

3) Construction of the infinite series for the solution

The solution $T(x, t)$ is assumed to be expressed by the infinite series

$$
T(x, t)=\sum_{i=1}^{\infty} u_{i}(x, t)
$$

where $u_{i}$ is selected from the candidate temperature functions of $U_{\mathrm{R}}\left( \pm T_{1}, a, x, t\right), U_{\mathrm{L}}\left( \pm T_{1}, b, x, t\right), U_{\mathrm{R}}\left( \pm T_{2}, a^{\prime}, x, t\right)$ and $U_{\mathrm{L}}\left( \pm T_{2}, b^{\prime}, x, t\right)$ with various values of $a, b, a^{\prime}$ and $b^{\prime}$. It is apparent that each $u_{i}$ and the partial sum of $N$ terms satisfy the heat conduction equation of Eq. (3.3-1)a and the initial condition of Eq. (3.3-1)b. The temperature function and the value of $a, b, a^{\prime}$ or $b^{\prime}$ for each $u_{i}$ are determined so that the errors of the partial sum of $N$ terms at both surfaces converge to zero as $N$ increases to infinity. Then the infinite series would satisfy the initial-boundary value problem of Eqs. (3.3-1)a - (3.3-1)c.

The first term $u_{1}$ of the series is determined so that the value of $u_{1}$ is equal to $T_{1}$ at $x=-L$. Then $u_{1}$ is set as

$$
u_{1}=U_{R}\left(T_{1},-L, x, t\right)=T_{1} \operatorname{erfc}\left(\frac{L+x}{2 \sqrt{\alpha t}}\right)
$$

The errors of $u_{1}$ at $x=-L$ and $x=L$ are 0 and $T_{1} \operatorname{erfc}(2 L /(2 \sqrt{\alpha t}))-T_{2}$, respectively. $u_{1}$ is shown in Fig. 3 .

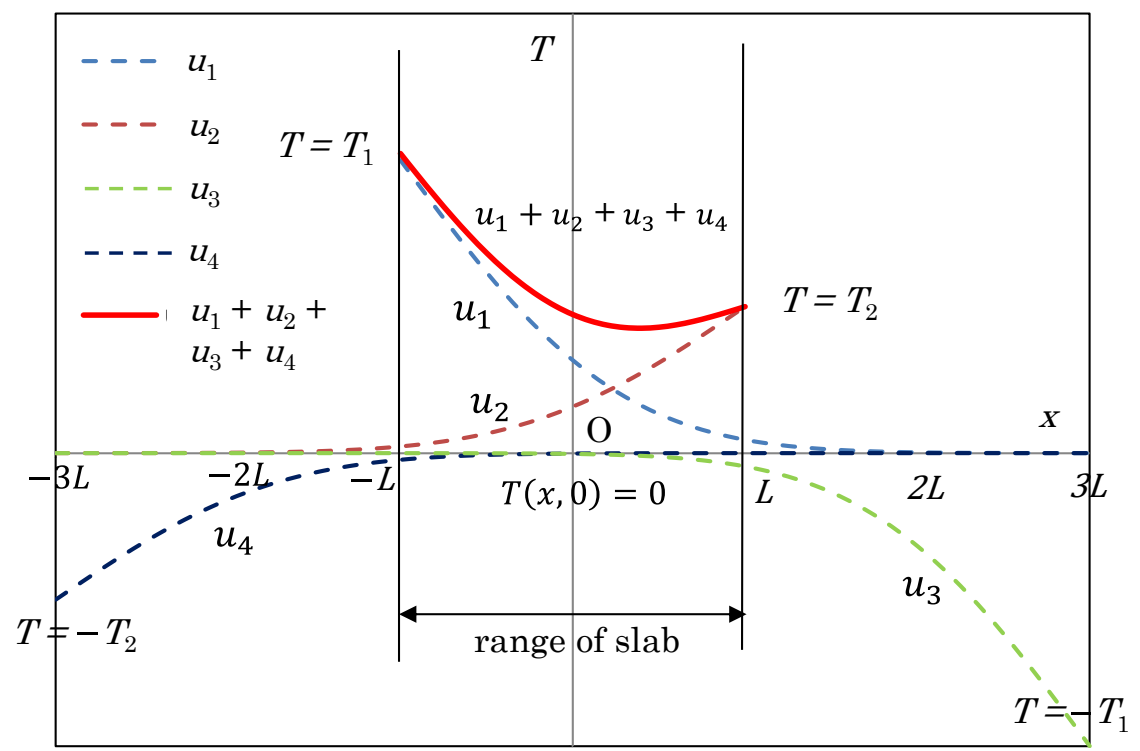

Fig. $3 u_{1}, u_{2}, u_{3}, u_{4}$ and $u_{1}+u_{2}+u_{3}+u_{4}$ (Example 3$)$ 
The second term $u_{2}$ is determined so that the value of $u_{1}+u_{2}$ is equal to $u_{1}(L, t)+T_{2}$ at $x=L$. Then $u_{2}$ is set as

$$
u_{2}=U_{L}\left(T_{2}, L, x, t\right)=T_{2} \operatorname{erfc}\left(\frac{L-x}{2 \sqrt{\alpha t}}\right)
$$

The errors of $u_{1}+u_{2}$ at $x=-L$ and $x=L$ are $T_{2} \operatorname{erfc}(2 L /(2 \sqrt{\alpha t}))$ and $T_{1} \operatorname{erfc}(2 L /(2 \sqrt{\alpha t}))$, respectively.

In the similar way, $u_{3}, u_{4}, u_{5},----u_{8}$ are set as

$$
\begin{gathered}
u_{3}=U_{L}\left(-T_{1}, 3 L, x, t\right)=-U_{L}\left(T_{1}, 3 L, x, t\right)=-T_{1} \operatorname{erfc}((3 L-x) /(2 \sqrt{\alpha t})) \\
u_{4}=U_{R}\left(-T_{2},-3 L, x, t\right)=-U_{R}\left(T_{2},-3 L, x, t\right)=-T_{2} \operatorname{erfc}((3 L+x) /(2 \sqrt{\alpha t})) \\
u_{5}=U_{R}\left(T_{1},-5 L, x, t\right)=T_{1} \operatorname{erfc}((5 L+x) /(2 \sqrt{\alpha t})) \\
u_{6}=U_{L}\left(T_{1}, 5 L, x, t\right)=T_{2} \operatorname{erfc}((5 L-x) /(2 \sqrt{\alpha t})) \\
u_{7}=U_{L}\left(-T_{1}, 7 L, x, t\right)=-U_{L}\left(T_{1}, 7 L, x, t\right)=-T_{1} \operatorname{erfc}((7 L-x) /(2 \sqrt{\alpha t})) \\
u_{8}=U_{R}\left(-T_{2},-7 L, x, t\right)=-U_{R}\left(T_{2},-7 L, x, t\right)=-T_{2} \operatorname{erfc}((7 L+x) /(2 \sqrt{\alpha t}))
\end{gathered}
$$

The errors for $\sum_{I=1}^{3} u_{i}, \sum_{I=1}^{4} u_{i}, \sum_{I=1}^{5} u_{i}, \sum_{I=1}^{6} u_{i}, \sum_{I=1}^{7} u_{i}, \sum_{I=1}^{8} u_{i}$ at $x=-L$ are $T_{2} \operatorname{erfc}(2 L /(2 \sqrt{\alpha t}))$ $-T_{1} \operatorname{erfc}(4 L /(2 \sqrt{\alpha t})),-T_{1} \operatorname{erfc}(4 L /(2 \sqrt{\alpha t})), 0, T_{2} \operatorname{erfc}(6 L /(2 \sqrt{\alpha t})), T_{2} \operatorname{erfc}(6 L /(2 \sqrt{\alpha t}))-T_{1} \operatorname{erfc}(8 L /(2 \sqrt{\alpha t}))$, and $-T_{1} \operatorname{erfc}(8 L /(2 \sqrt{\alpha t}))$, respectively. The errors at $x=L$ are $0,-T_{2} \operatorname{erfc}(4 L /(2 \sqrt{\alpha t})),-T_{2} \operatorname{erfc}(4 L /$ $(2 \sqrt{\alpha t}))+T_{1} \operatorname{erfc}(6 L /(2 \sqrt{\alpha t})), T_{1} \operatorname{erfc}(6 L /(2 \sqrt{\alpha t})), 0$, and $-T_{2} \operatorname{erfc}(8 L /(2 \sqrt{\alpha t}))$, respectively. It is seen that the errors for $\sum_{I=1}^{N} u_{i}$ at $x= \pm L$ converge to zero as the number of terms $N$ increases to infinity.

The temperature functions $u_{1}, u_{2} u_{3}, u_{4}$ and the sum $u_{1}+u_{2}+u_{3}+u_{4}$ are shown schematically in Fig 3 . Thus, the solution becomes

$$
\begin{aligned}
T(x, t)= & U_{R}\left(T_{1},-L, x, t\right)+U_{L}\left(T_{2}, L, x, t\right)+U_{L}\left(-T_{1}, 3 L, x, t\right)+U_{R}\left(-T_{2},-3 L, x, t\right)+--- \\
= & \sum_{n=0}^{\infty}\left(T_{1} \operatorname{erfc} \frac{(4 n+1) L+x}{2 \sqrt{\alpha t}}+T_{2} \operatorname{erfc} \frac{(4 n+1) L-x}{2 \sqrt{\alpha t}}\right. \\
& \left.-T_{1} \operatorname{erfc} \frac{(4 n+3) L-x}{2 \sqrt{\alpha t}}-T_{2} \operatorname{erfc} \frac{(4 n+3) L+x}{2 \sqrt{\alpha t}}\right) \\
= & T_{1} \sum_{n=0}^{\infty} \operatorname{erfc} \frac{(4 n+1) L+x}{2 \sqrt{\alpha t}}+T_{2} \sum_{n=0}^{\infty} \operatorname{erfc} \frac{(4 n+1) L-x}{2 \sqrt{\alpha t}} \\
= & T_{1} \sum_{n=0}^{\infty}\left(\operatorname{erfc} \frac{(4 n+1) L+x}{2 \sqrt{\alpha t}}-\operatorname{erfc} \frac{(4 n+3) L-x}{2 \sqrt{\alpha t}}\right)+T_{2} \sum_{n=0}^{\infty}\left(\operatorname{erfc} \frac{(4 n+1) L-x}{2 \sqrt{\alpha t}}-\operatorname{erfc} \frac{(4 n+3) L+x}{2 \sqrt{\alpha t}}\right)
\end{aligned}
$$

\subsubsection{Method2}

Here, the solution of Eqs. (3.3-1)a - (3.3-1)c is obtained directly by using the results of Examples 1 and 2 . We consider a slab of thickness $2 L$ with zero initial temperature and the edge surfaces at $x=-L$ and $x=L$ kept at constant 
temperature $T_{1}$ and zero, respectively. Changing the origin in Eq. (3.1-10) to the middle point of the slab and replacing $L$ by $2 L$ and $T_{0}$ by $T_{1}$, we obtain

$$
T(x, t)=T_{1} \sum_{n=0}^{\infty}\left(\operatorname{erfc} \frac{(4 n+1) L+x}{2 \sqrt{\alpha t}}-\operatorname{erfc} \frac{(4 n+3) L-x}{2 \sqrt{\alpha t}}\right)
$$

In the same way, the solution of a slab with zero initial temperature and the edge surfaces at $x=-L$ and $x=L$ kept at zero and constant temperature $T_{2}$, respectively, is

$$
T(x, t)=T_{2} \sum_{n=0}^{\infty}\left(\operatorname{erfc} \frac{(4 n+1) L-x}{2 \sqrt{\alpha t}}-\operatorname{erfc} \frac{(4 n+3) L+x}{2 \sqrt{\alpha t}}\right)
$$

The sum of Eqs. (3.3-10) and (3.3-11) becomes the solution for the initial-boundary value problem of Eqs. (3.3-1)a (3.3-1)c, which is expressed by

$$
\begin{aligned}
T(x, t)=T_{1} \sum_{n=0}^{\infty}\left(\operatorname{erfc} \frac{(4 n+1) L+x}{2 \sqrt{\alpha t}}\right. & -\operatorname{erfc} \frac{(4 n+3) L-x}{2 \sqrt{\alpha t})} \\
+ & T_{2} \sum_{n=0}^{\infty}\left(\operatorname{erfc} \frac{(4 n+1) L-x}{2 \sqrt{\alpha t}}-\operatorname{erfc} \frac{(4 n+3) L+x}{2 \sqrt{\alpha t}}\right)
\end{aligned}
$$

This formula of the solution is the same as Eq. (3.3-9) by Method 1.

When $T_{1}=T_{2}$, the solution becomes

$$
\begin{aligned}
T(x, t)=T_{1} \sum_{n=0}^{\infty}\left(\operatorname{erfc} \frac{(4 n+1) L+x}{2 \sqrt{\alpha t}}+\operatorname{erfc}\right. & \frac{(4 n+1) L-x}{2 \sqrt{\alpha t}} \\
& \left.-\operatorname{erfc} \frac{(4 n+3) L+x}{2 \sqrt{\alpha t}}-\operatorname{erfc} \frac{(4 n+3) L-x}{2 \sqrt{\alpha t}}\right)
\end{aligned}
$$

Eq. (3.3-13) can be also expressed by

$$
T(x, t)=T_{1} \sum_{n=0}^{\infty}(-1)^{n}\left(\operatorname{erfc} \frac{(2 n+1) L+x}{2 \sqrt{\alpha t}}+\operatorname{erfc} \frac{(2 n+1) L-x}{2 \sqrt{\alpha t}}\right)
$$

\subsection{Example 4 (A slab $0 \leq x \leq L$ with zero initial temperature, and the surfaces at $x=0$ insulated and at $x=L$ maintained at constant temperature $T_{0}$ )}

The heat conduction problem of Example 4 is shown in Fig. 4. The solution of Example 3 defined in $-L \leq x \leq$ $L$ in the case of $T_{1}=T_{2}$, Eq. (3.3-14), is symmetric with respect to the line of $x=0$. This means that the heat flow is zero at $x=0$. (The derivative of $T$ with respect to $x$ at $x=0$ is zero or insulated.) It is also seen from Eq. (3.3-14) that the temperature at $x=L$ is $T_{1}$. When $T_{1}$ is replaced by $T_{0}$ and the definition region of $x$ is set between 0 and $L$, Eq. (3.3-14) becomes the solution of Example 4, which is expressed by

$$
\begin{aligned}
T(x, t) & =T_{0} \sum_{n=0}^{\infty}(-1)^{n}\left(\operatorname{erfc} \frac{(2 n+1) L+x}{2 \sqrt{\alpha t}}+\operatorname{erfc} \frac{(2 n+1) L-x}{2 \sqrt{\alpha t}}\right) \\
& =T_{0} \sum_{n=0}^{\infty}(-1)^{n} \operatorname{erfc} \frac{(2 n+1) L+x}{2 \sqrt{\alpha t}}+T_{0} \sum_{n=0}^{\infty}(-1)^{n} \operatorname{erfc} \frac{(2 n+1) L-x}{2 \sqrt{\alpha t}}
\end{aligned}
$$

This formula is the same as that which was solved by Laplace transformation for the equivalent heat conduction problem for a slab (Carslaw and Jaeger, 1959). 


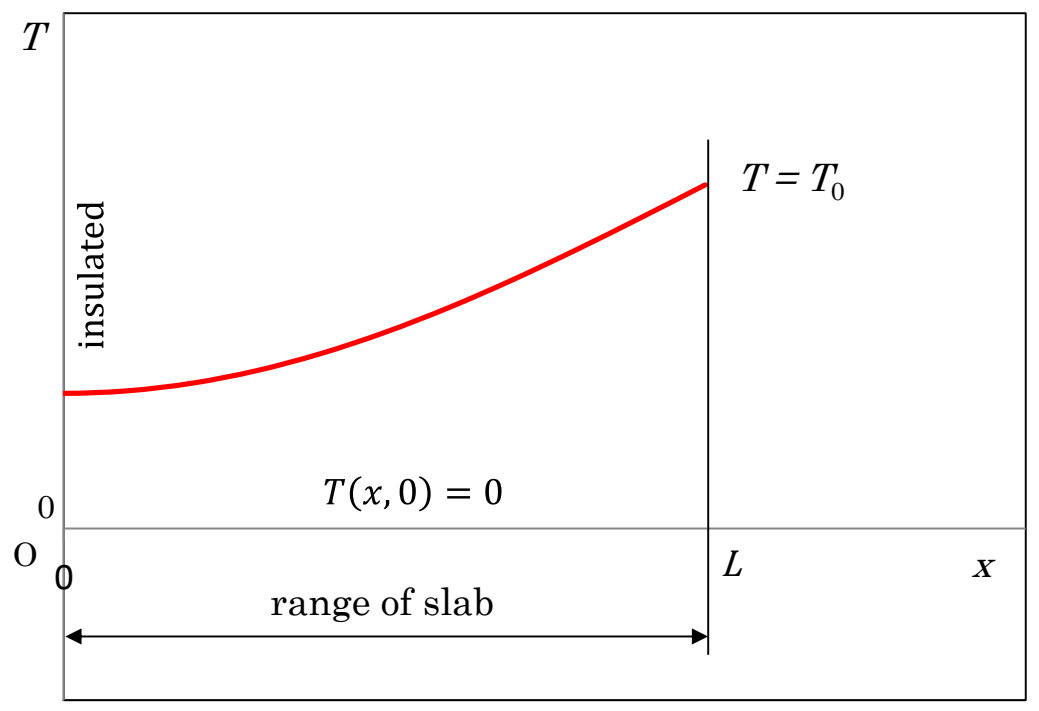

Fig. 4 Heat conduction problem of Example 4

3.5 Example 5 (A slab $-L \leq x \leq L$ with constant initial temperature $T_{\mathrm{i}}(\neq 0)$ and two edge surfaces of $x=-L$ and $x=L$ kept at constant temperatures of $T_{1}$ and $T_{2}$, respectively)

The initial-boundary value problem is expressed as

$$
\begin{gathered}
\frac{\partial T}{\partial t}=\alpha \frac{\partial^{2} T}{\partial x^{2}} \quad(-L<x<L, t>0) \\
T(x, 0)=T_{\mathrm{i}} \quad(-L<x<L) \\
T(-L, t)=T_{1}, \quad T(L, t)=T_{2} \quad(t>0)
\end{gathered}
$$

Eqs. (3.5-1)a - (3.5-1)c are the same as Eqs. (3.3-1)a - (3.3-1)c except for the value of $T_{\mathrm{i}}$ (Example 3: $T_{\mathrm{i}}=0$, Example 5: $\left.T_{\mathrm{i}} \neq 0\right)$. If we put

$$
T(x, t)=T_{\mathrm{i}}+U(x, t)
$$

and substitute Eq. (3.5-2) into Eqs. (3.5-1)a - (3.5-1)c, we obtain the following initial-boundary value problem

$$
\begin{gathered}
\frac{\partial U}{\partial t}=\alpha \frac{\partial^{2} U}{\partial x^{2}} \quad(-L<x<L, t>0) \\
U(x, 0)=0 \quad(-L<x<L) \\
U(-L, t)=T_{1}-T_{i}, \quad U(L, t)=T_{2}-T_{i} \quad(t>0)
\end{gathered}
$$

By replacing $T_{1}$ and $T_{2}$ by $T_{1}-T_{\mathrm{i}}$, and $T_{2}-T_{\mathrm{i}}$, respectively, in Eq. (3.3-12), and using Eq. (3.5-2), we obtain

$$
\begin{aligned}
T(x, t)=T_{\mathrm{i}}+\left(T_{1}-T_{\mathrm{i}}\right) \sum_{n=0}^{\infty}\left(\operatorname{erfc} \frac{(4 n+1) L+x}{2 \sqrt{\alpha t}}-\operatorname{erfc} \frac{(4 n+3) L-x}{2 \sqrt{\alpha t}}\right) \\
\quad+\left(T_{2}-T_{\mathrm{i}}\right) \sum_{n=0}^{\infty}\left(\operatorname{erfc} \frac{(4 n+1) L-x}{2 \sqrt{\alpha t}}-\operatorname{erfc} \frac{(4 n+3) L+x}{2 \sqrt{\alpha t}}\right)
\end{aligned}
$$

As stated above, when constant $T_{\mathrm{i}}$ is not zero, the problem is transformed to that with zero initial temperature. Then we can solve the problem by the method of this paper.

3.6 Example 6 (A slab of thickness $L(0 \leq x \leq L)$ with zero initial temperature and two edge surfaces kept insulated at $x=0$ and at constant heat flux $F_{0}$ at $x=L$ )

1) Definition of the heat conduction problem in a slab

Consider the problem of heat flow in a slab $0 \leq x \leq L$ with the boundary surfaces of $x=0$ insulated and $x=L$ kept 
at constant heat flux of $F_{0}$, when the initial temperature is zero throughout. Then the initial-boundary value problem is

$$
\begin{aligned}
& \frac{\partial T}{\partial t}=\alpha \frac{\partial^{2} T}{\partial x^{2}} \quad(0<x<L, t>0) \\
& T(x, 0)=0 \quad(0<x<L) \\
& -k T_{x}(0, t)=0\left(\text { or } T_{x}(0, t)=0\right), \quad-k T_{x}(L, t)=-F_{0} \quad(t>0)
\end{aligned}
$$

2) Definition of the temperature functions in semi-infinite solids

We consider the temperature distribution in a semi-infnite solid defined in $a \leq x<\infty$ with zero initial temperature, where the surface is located at $x=a$ and kept at constant heat flux $F_{0}$. The initial-boundary value problem for the temperature function $u(x, t)=U_{\mathrm{R}}\left(F_{0}, a, x, t\right)$ is

$$
\begin{array}{cc}
\frac{\partial u}{\partial t}=\alpha \frac{\partial^{2} u}{\partial x^{2}} & (x>a, t>0) \\
u(x, 0)=0 & (x>a) \\
-k u_{x}(a, t)=F_{0} & (t>0) \\
u(x, t) \rightarrow 0 \quad \text { as } \quad x \rightarrow \infty & (t>0)
\end{array}
$$

(Eqs. (3.6-2)a - (3.6-2)d for $a=0$ are given by Churchill (1958).) The solution for the problem is

$$
\begin{aligned}
u(x, t)=U_{R}\left(F_{0}, a, x, t\right)= & \frac{F_{0}}{k}\left[2 \sqrt{\frac{\alpha t}{\pi}} e^{-(x-a)^{2} /(4 \alpha t)}-(x-a) \operatorname{erfc} \frac{x-a}{2 \sqrt{\alpha t}}\right] \\
& =2 \sqrt{\alpha t} \frac{F_{0}}{k} \operatorname{ierfc} \frac{x-a}{2 \sqrt{\alpha t}}
\end{aligned}
$$

(Eq. (3.6-3)a for $a=0$ is given by Churchill (1958) and Incropera et al. (2007). Eqs. (3.6-3)a and (3.6-3)b for $a=0$ are given by Carslaw and Jaeger (1959).) In the similar way, $U_{\mathrm{L}}\left(F_{0}, b, x, t\right)$ is defined to be the solution of the following initial-boundary value problem, where the solid is defined in $-\infty<x \leq b$

$$
\begin{aligned}
& \frac{\partial u}{\partial t}=\alpha \frac{\partial^{2} u}{\partial x^{2}}(x<b, t>0) \\
& u(x, 0)=0(x<b) \\
&-k u_{x}(b, t)=-F_{0}(t>0) \\
& u(x, t) \rightarrow 0 \quad \text { as } \quad x \rightarrow-\infty \quad(t>0)
\end{aligned}
$$

The solution for the problem is

$$
\begin{aligned}
u(x, t)=U_{L}\left(F_{0}, b, x, t\right) & =\frac{F_{0}}{k}\left[2 \sqrt{\frac{\alpha t}{\pi}} e^{-(x-b)^{2} /(4 \alpha t)}-(b-x) \operatorname{erfc} \frac{b-x}{2 \sqrt{\alpha t}}\right] \\
& =2 \sqrt{\alpha t} \frac{F_{0}}{k} \text { ierfc } \frac{b-x}{2 \sqrt{\alpha t}}
\end{aligned}
$$

$U_{\mathrm{R}}\left(F_{0}, a, x, t\right)$ is symmetric with $U_{\mathrm{L}}\left(F_{0}, b, x, t\right)$ with respect to the line of $x=(a+b) / 2$ and $\partial\left(U_{\mathrm{R}}+U_{\mathrm{L}}\right) / \partial x=0$ at $x=$ $(a+b) / 2$ with $a \leq b . U_{\mathrm{R}}\left(F_{0},-a, x, t\right)$ is symmetric with $U_{\mathrm{L}}\left(F_{0}, a, x, t\right)$ with respect to the line of $x=0$ and $\partial\left(U_{\mathrm{R}}+U_{\mathrm{L}}\right) / \partial x=0$ at $x=0$ with $a \geq 0$.

The partial derivatives with respect to $x$ are

$$
\begin{aligned}
& \frac{\partial U_{R}\left(F_{0}, a, x, t\right)}{\partial x}=-\frac{F_{0}}{k} \operatorname{erfc} \frac{x-a}{2 \sqrt{\alpha t}} \\
& \frac{\partial U_{L}\left(F_{0}, b, x, t\right)}{\partial x}=\frac{F_{0}}{k} \operatorname{erfc} \frac{b-x}{2 \sqrt{\alpha t}}
\end{aligned}
$$

3) Construction of the infinite series for the solution

The solution $T(x, t)$ is assumed to be expressed by the infinite series 


$$
T(x, t)=\sum_{i=1}^{\infty} u_{i}(x, t)
$$

where $u_{i}$ is selected from the temperature functions of $U_{\mathrm{R}}\left(F_{0}, a, x, t\right)$ and $U_{\mathrm{L}}\left(F_{0}, b, x, t\right)$ with various values of $a$ and $b$. It is apparent that each $u_{i}$ and the partial sum of $N$ terms satisfy the heat conduction equation of Eq. (3.6-1)a and the initial condition of Eq. (3.6-1)b. The temperature function and the value of $a$ or $b$ for each $u_{i}$ are determined so that the derivatives of the partial sum of $N$ terms with respect to $x$ multiplied by $-k$ at $x=0$ and $x=L$ converge to zero and $-F_{0}$, respectively, as $N$ increases to infinity. Then the infinite series would satisfy the initial-boundary value problem of Eqs. (3.6-1)a - (3.6-1)c.

Based on the above consideration, the first term $u_{1}$ is determined so that it satisfies the boundary condition (constant heat flux) at $x=L$. Then $u_{1}$ is set as

$$
\begin{aligned}
u_{1}=U_{L}\left(F_{0}, L, x, t\right)= & \frac{F_{0}}{k}\left[2 \sqrt{\frac{\alpha t}{\pi}} e^{-(L-x)^{2} /(4 \alpha t)}-(L-x) \operatorname{erfc} \frac{L-x}{2 \sqrt{\alpha t}}\right] \\
& =2 \sqrt{\alpha t} \frac{F_{0}}{k} \operatorname{ierfc} \frac{L-x}{2 \sqrt{\alpha t}}
\end{aligned}
$$

The values of $-k \partial u_{1} / \partial x$ at $x=0$ and $x=L$ are $-F_{0} \operatorname{erfc}(L /(2 \sqrt{\alpha t}))$ and $-F_{0}$, respectively. This means that the errors of heat flux at $x=0$ and $x=L$ are $-F_{0} \operatorname{erfc}(L /(2 \sqrt{\alpha t}))$ and 0 , respectively. $u_{1}$ is shown schematically in Fig. 5 .

The second term $u_{2}$ is determined so that $u_{1}+u_{2}$ satisfies the boundary condition (zero heat flux) at $x=0$. Then $u_{2}$ is set as

$$
\begin{aligned}
u_{2}=U_{R}\left(F_{0},-L, x, t\right)= & \frac{F_{0}}{k}\left[2 \sqrt{\frac{\alpha t}{\pi}} e^{-(L+x)^{2} /(4 \alpha t)}-(L+x) \operatorname{erfc} \frac{L+x}{2 \sqrt{\alpha t}}\right] \\
& =2 \sqrt{\alpha t} \frac{F_{0}}{k} \operatorname{ierfc} \frac{L+x}{2 \sqrt{\alpha t}}
\end{aligned}
$$

The values of $-k \partial\left(u_{1}+u_{2}\right) / \partial x$ at $x=0$ and $x=L$ are zero and $-F_{0}+F_{0} \operatorname{erfc}(2 L /(2 \sqrt{\alpha t}))$, respectively. This means that the errors of heat flux at $x=0$ and $x=L$ are 0 and $F_{0} \operatorname{erfc}(2 L /(2 \sqrt{\alpha t}))$, respectively. $u_{2}$ is shown schematically in Fig. 5.

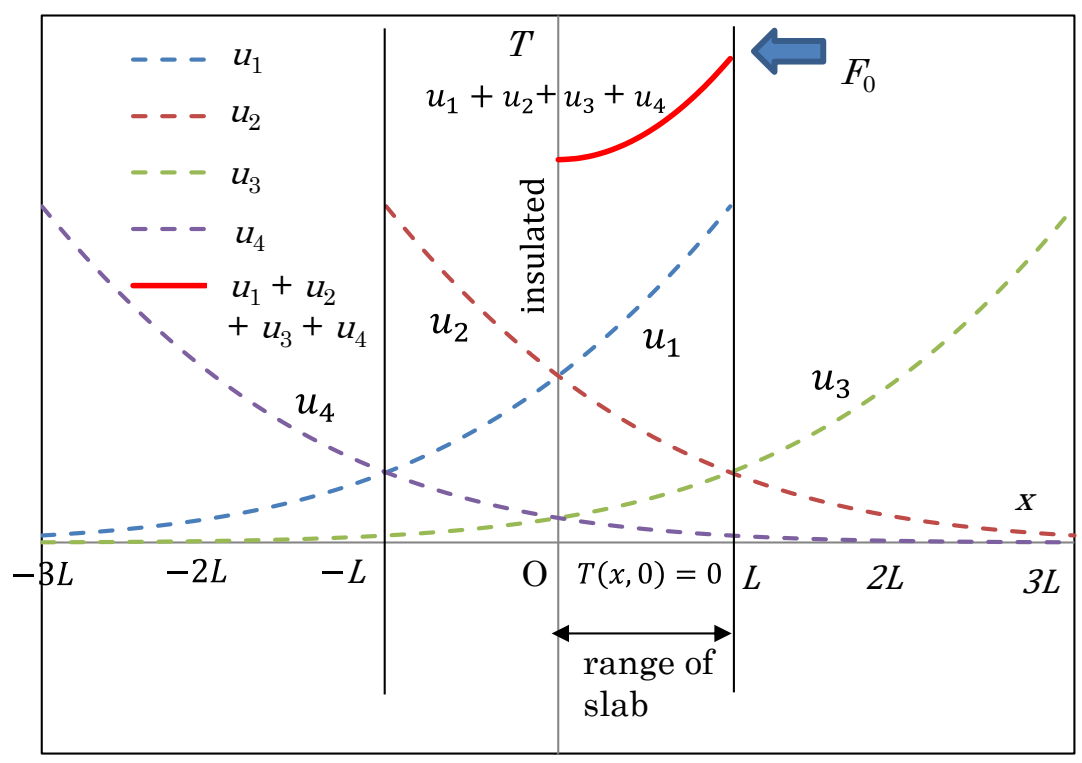

Fig. $5 u_{1}, u_{2}, u_{3}, u_{4}$ and $u_{1}+u_{2}+u_{3}+u_{4}$ (Example 6)

The third term $u_{3}$ is determined so that $u_{1}+u_{2}+u_{3}$ satisfies the boundary condition at $x=L$. Then $u_{3}$ is set as 


$$
\begin{aligned}
u_{3}=U_{L}\left(F_{0}, 3 L, x, t\right) & =\frac{F_{0}}{k}\left[2 \sqrt{\frac{\alpha t}{\pi}} e^{-(3 L-x)^{2} /(4 \alpha t)}-(3 L-x) \operatorname{erfc} \frac{3 L-x}{2 \sqrt{\alpha t}}\right] \\
& =2 \sqrt{\alpha t} \frac{F_{0}}{k} \text { ierfc } \frac{3 L-x}{2 \sqrt{\alpha t}}
\end{aligned}
$$

Then the values of $-k \partial\left(u_{1}+u_{2}+u_{3}\right) / \partial x$ at $x=0$ and $x=L$ are $-F_{0} \operatorname{erfc}(3 L /(2 \sqrt{\alpha t}))$ and $-F_{0}$, respectively. This means that the errors of heat flux at $x=0$ and $x=L$ are $-F_{0} \operatorname{erfc}(3 L /(2 \sqrt{\alpha t}))$ and 0 , respectively. $u_{3}$ is shown schematically in Fig. 5.

The forth term $u_{4}$ is determined so that $u_{1}+u_{2}+u_{3}+u_{4}$ satisfies the boundary condition at $x=0$. Then $u_{4}$ is set as

$$
\begin{aligned}
u_{4}=U_{R}\left(F_{0},-3 L, x, t\right) & =\frac{F_{0}}{k}\left[2 \sqrt{\frac{\alpha t}{\pi}} e^{-(3 L+x)^{2} /(4 \alpha t)}-(3 L+x) \operatorname{erfc} \frac{3 L+x}{2 \sqrt{\alpha t}}\right] \\
& =2 \sqrt{\alpha t} \frac{F_{0}}{k} \operatorname{ierfc} \frac{3 L+x}{2 \sqrt{\alpha t}}
\end{aligned}
$$

The values of $-k \partial\left(u_{1}+u_{2}+u_{3}+u_{4}\right) / \partial x$ at $x=0$ and $x=L$ are zero and $-F_{0}+F_{0} \operatorname{erfc}(4 L /(2 \sqrt{\alpha t}))$, respectively. This means that the errors of heat flux at $x=0$ and $x=L$ are 0 and $F_{0} \operatorname{erfc}(4 L /(2 \sqrt{\alpha t}))$, respectively. $u_{4}$ and $u_{1}+u_{2}+u_{3}$ $+u_{4}$ are shown schematically in Fig. 5 .

In the same way, other terms can be determined. The maximum absolute error of heat flux for the partial sum of $N$ terms at the surfaces is $F_{0} \operatorname{erfc}(N L /(2 \sqrt{\alpha t}))$, which decreases monotonically and converges to zero as $N \rightarrow \infty$. Thus the formula for the solution of Eqs. (3.6-1)a - (3.6-1)c becomes

$$
T(x, t)=\frac{2 F_{0} \sqrt{\alpha t}}{k} \sum_{n=0}^{\infty}\left(\operatorname{ierfc} \frac{(2 n+1) L-x}{2 \sqrt{\alpha t}}+\operatorname{ierfc} \frac{(2 n+1) L+x}{2 \sqrt{\alpha t}}\right)
$$

This formula of the solution is the same as that for the same heat conduction problem, which was obtained by the method of Laplace transformation (Carslaw and Jaeger, 1959).

\subsection{Example 7 (A slab of thickness $L(0 \leq x \leq L)$ with zero initial temperature and the edge surfaces of $x=0$ kept at zero temperature and $x=L$ at constant heat flux $F_{0}$ )}

1) Definition of the heat conduction problem in a slab

The problem is the same as that of Example 6 except that the boundary surface at $x=0$ for Example 7 is at zero temperature instead of "insulated". The initial-boundary value problem is

$$
\begin{array}{cl}
\frac{\partial T}{\partial t}=\alpha \frac{\partial^{2} T}{\partial x^{2}} & (0<x<L, t>0) \\
T(x, 0)=0 & (0<x<L) \\
T(0, t)=0, \quad-k T_{x}(L, t)=-F_{0} \quad(t>0)
\end{array}
$$

2) Definition of the temperature functions in semi-infinite solids

The following temperature functions are used

$$
\begin{aligned}
U_{R}\left(F_{0}, a, x, t\right) & =\frac{F_{0}}{k}\left[2 \sqrt{\frac{\alpha t}{\pi}} e^{-(x-a)^{2} /(4 \alpha t)}-(x-a) \operatorname{erfc} \frac{x-a}{2 \sqrt{\alpha t}}\right] \\
& =\frac{2 F_{0} \sqrt{\alpha t}}{k} \operatorname{ierfc} \frac{x-a}{2 \sqrt{\alpha t}} \\
U_{L}\left(F_{0}, b, x, t\right) & =\frac{F_{0}}{k}\left[2 \sqrt{\frac{\alpha t}{\pi}} e^{-(b-x)^{2} /(4 \alpha t)}-(b-x) \operatorname{erfc} \frac{b-x}{2 \sqrt{\alpha t}}\right]
\end{aligned}
$$




$$
=\frac{2 F_{0} \sqrt{\alpha t}}{k} \operatorname{ierfc} \frac{b-x}{2 \sqrt{\alpha t}}
$$

These temperature functions are the same as those (Eqs. (3.6-3)a, (3.6-3)b, (3.6-5)a, and (3.6-5)b) in Example 6. In addition, $U_{\mathrm{R}}\left(-F_{0}, a, x, t\right)\left(=-U_{\mathrm{R}}\left(F_{0}, a, x, t\right)\right)$ and $U_{\mathrm{L}}\left(-F_{0}, b, x, t\right)\left(=-U_{\mathrm{L}}\left(F_{0}, b, x, t\right)\right)$ are also set as the temperature functions for the construction of the solution.

3) Construction of the infinite series for the solution

The solution $T(x, t)$ is assumed to be expressed by the infinite series

$$
T(x, t)=\sum_{i=1}^{\infty} u_{i}(x, t)
$$

where $u_{i}$ is selected from the candidate temperature functions of $U_{\mathrm{R}}\left( \pm F_{0}, a, x, t\right)$ and $U_{\mathrm{L}}\left( \pm F_{0}, b, x, t\right)$ with various values of $a$ and $b$. It is apparent that $u_{i}$ satisfies the heat conduction equation of Eq. (3.7-1)a and the initial condition of Eq. (3.7-1)b. Then the partial sum of $N$ terms also satisfies the same Eqs. (3.7-1)a and (3.7-1)b. The temperature function and the value of $a$ or $b$ for each $u_{i}$ are determined so that the partial sum of $N$ terms at $x=0$ converges to zero and the derivative of the partial sum with respect to $x$ at $x=L$ multiplied by $-k$ converges to $-F_{0}$, as $N$ increases to infinity. Then the infinite series would satisfy the initial-boundary value problem of Eqs. (3.7-1)a - (3.7-1)c.

The first term $u_{1}$ is determined so that the boundary condition at $x=L$ is satisfied. Then $u_{1}$ is set as

$$
\begin{aligned}
u_{1}=U_{L}\left(F_{0}, L, x, t\right) & =\frac{F_{0}}{k}\left[2 \sqrt{\frac{\alpha t}{\pi}} e^{-(L-x)^{2} /(4 \alpha t)}-(L-x) \operatorname{erfc} \frac{L-x}{2 \sqrt{\alpha t}}\right] \\
& =\frac{2 F_{0} \sqrt{\alpha t}}{k} \operatorname{ierfc} \frac{L-x}{2 \sqrt{\alpha t}}
\end{aligned}
$$

The value of $u_{1}$ at $x=0$ is $\left(2 \sqrt{\alpha t} F_{0} / k\right) \operatorname{ierfc}(L /(2 \sqrt{\alpha t}))$, and $-k \partial u_{1} / \partial x$ at $x=L$ is $-F_{0}$. The errors of $u_{1}$ and $-k \partial u_{1} / \partial x$ at $x=0$ and $x=L$ are $\left(2 \sqrt{\alpha t} F_{0} / k\right) \operatorname{ierfc}(L /(2 \sqrt{\alpha t}))$ and zero, respectively. $u_{1}$ is shown schematically in Fig. 6.

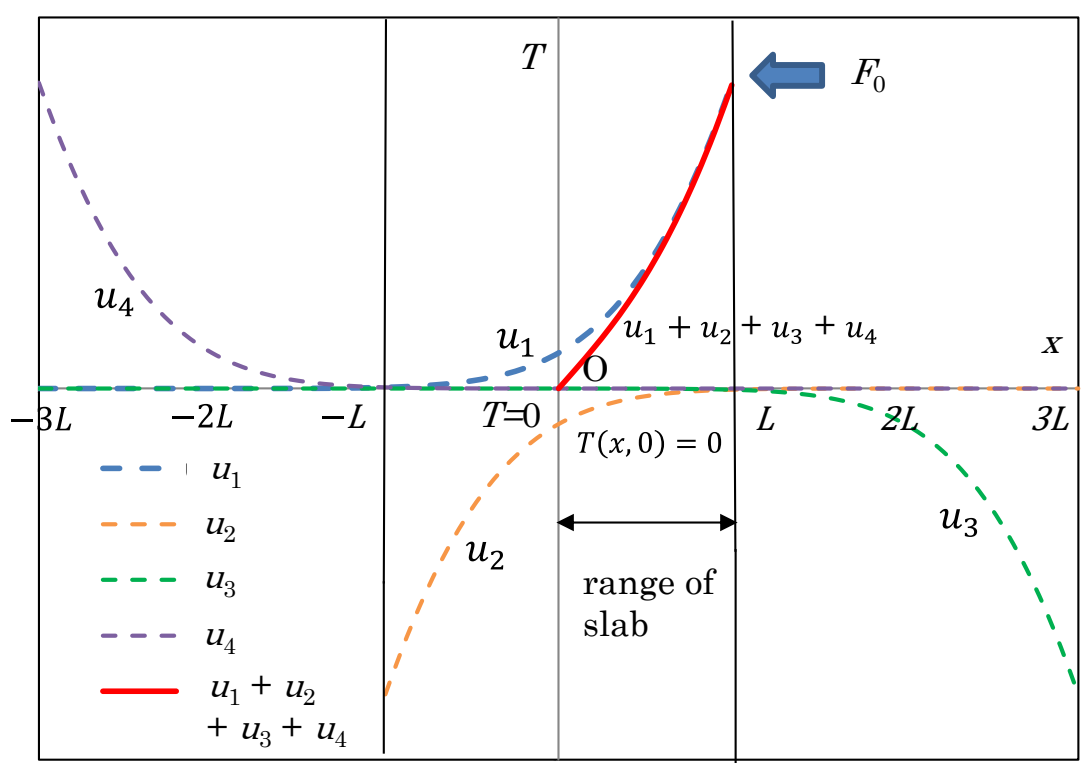

Fig. $6 u_{1}, u_{2}, u_{3}, u_{4}$ and $u_{1}+u_{2}+u_{3}+u_{4}$ (Example 7)

The second term $u_{2}$ is determined so that $u_{1}+u_{2}$ satisfies the boundary condition at $x=0$. Then $u_{2}$ is set as 


$$
\begin{aligned}
u_{2}=U_{R}\left(-F_{0},-L, x, t\right) & =-\frac{F_{0}}{k}\left[2 \sqrt{\frac{\alpha t}{\pi}} e^{-(L+x)^{2} /(4 \alpha t)}-(L+x) \operatorname{erfc} \frac{L+x}{2 \sqrt{\alpha t}}\right] \\
& =-\frac{2 F_{0} \sqrt{\alpha t}}{k} \operatorname{ierfc} \frac{L+x}{2 \sqrt{\alpha t}}
\end{aligned}
$$

The value of $u_{1}+u_{2}$ at $x=0$ is zero and $-k \partial\left(u_{1}+u_{2}\right) / \partial x$ at $x=L$ is $-F_{0}-F_{0} \operatorname{erfc}(2 L /(2 \sqrt{\alpha t}))$. The errors of $u_{1}+$ $u_{2}$ at $x=0$ and $-k \partial\left(u_{1}+u_{2}\right) / \partial x$ at $x=L$ are zero and $-F_{0} \operatorname{erfc}(2 L /(2 \sqrt{\alpha t}))$, respectively. $u_{2}$ is shown schematically in Fig. 6.

The third term $u_{3}$ is determined so that $u_{1}+u_{2}+u_{3}$ satisfies the boundary condition at $x=L$. Then $u_{3}$ is set as

$$
\begin{aligned}
u_{3}=U_{L}\left(-F_{0}, 3 L, x, t\right) & =-\frac{F_{0}}{k}\left[2 \sqrt{\frac{\alpha t}{\pi}} e^{-(3 L-x)^{2} /(4 \alpha t)}-(3 L-x) \operatorname{erfc} \frac{3 L-x}{2 \sqrt{\alpha t}}\right] \\
= & -\frac{2 F_{0} \sqrt{\alpha t}}{k} \operatorname{ierfc} \frac{3 L-x}{2 \sqrt{\alpha t}}
\end{aligned}
$$

The value of $u_{1}+u_{2}+u_{3}$ at $x=0$ is $-\left(2 \sqrt{\alpha t} F_{0} / k\right) \operatorname{ierfc}(3 L /(2 \sqrt{\alpha t}))$, and $-k \partial\left(u_{1}+u_{2}+u_{3}\right) / \partial x$ at $x=L$ is $-F_{0}$. The errors of $u_{1}+u_{2}+u_{3}$ at $x=0$ and $-k \partial\left(u_{1}+u_{2}+u_{3}\right) / \partial x$ at $x=L$ are $-\left(2 \sqrt{\alpha t} F_{0} / k\right) \operatorname{ierfc}(3 L /(2 \sqrt{\alpha t}))$ and zero, respectively. $u_{3}$ is shown schematically in Fig. 6 .

The forth term $u_{4}$ is determined so that $u_{1}+u_{2}+u_{3}+u_{4}$ satisfies the boundary condition at $x=0$. Then $u_{4}$ is set as

$$
\begin{aligned}
u_{4}=U_{R}\left(F_{0},-3 L, x, t\right) & =\frac{F_{0}}{k}\left[2 \sqrt{\frac{\alpha t}{\pi}} e^{-(3 L+x)^{2} /(4 \alpha t)}-(3 L+x) \operatorname{erfc} \frac{3 L+x}{2 \sqrt{\alpha t}}\right] \\
& =\frac{2 F_{0} \sqrt{\alpha t}}{k} \operatorname{ierfc} \frac{3 L+x}{2 \sqrt{\alpha t}}
\end{aligned}
$$

The value of $u_{1}+u_{2}+u_{3}+u_{4}$ at $x=0$ is zero and $-k \partial\left(u_{1}+u_{2}+u_{3}+u_{4}\right) / \partial x$ at $x=L$ is $-F_{0}+F_{0} \operatorname{erfc}(4 L /$ $(2 \sqrt{\alpha t}))$. Then the errors of $u_{1}+u_{2}+u_{3}+u_{4}$ at $x=0$ and $-k \partial\left(u_{1}+u_{2}+u_{3}+u_{4}\right) / \partial x$ at $x=L$ are zero and $F_{0} \operatorname{erfc}(4 L /(2 \sqrt{\alpha t}))$, respectively. $u_{4}$ and $u_{1}+u_{2}+u_{3}+u_{4}$ are shown schematically in Fig. 6 .

In the same way, other terms can be determined. The errors of $\sum_{i=1}^{N} u_{i}$ at $x=0$ for $N=1,2$, ----- are

$$
\left(2 \sqrt{\alpha t} F_{0} / k\right) \operatorname{ierfc}(L /(2 \sqrt{\alpha t})), 0,-\left(2 \sqrt{\alpha t} F_{0} / k\right) \operatorname{ierfc}(3 L /(2 \sqrt{\alpha t})), 0,---
$$

and the errors of $-k \sum_{i=1}^{N} u_{i} / \partial x$ at $x=L$ for $N=1,2,----$ are

$$
0,-F_{0} \operatorname{erfc}(2 L /(2 \sqrt{\alpha t})), 0, F_{0} \operatorname{erfc}(4 L /(2 \sqrt{\alpha t})), 0,
$$

It is seen that both errors at $x=0$ and $x=L$ converge to zero as the number of terms increases to infinity. Thus the solution is

$$
T(x, t)=\frac{2 F_{0} \sqrt{\alpha t}}{k} \sum_{n=0}^{\infty}(-1)^{n}\left(\operatorname{ierfc} \frac{(2 n+1) L-x}{2 \sqrt{\alpha t}}-\operatorname{ierfc} \frac{(2 n+1) L+x}{2 \sqrt{\alpha t}}\right)
$$

This formula for the solution is the same as that of the solution for the same heat conduction problem solved by Laplace transformation (Carslaw and Jaeger, 1959).

\section{Validity of the solution method}

In this chapter the validity of the solution method is discussed. In the previous chapters we assumed that the solution with the infinite series representation obtained by the method proposed in this paper converges and satisfies the initial and boundary value problems (see Sec. 2.3). While it is necessary to prove this assumption from the mathematical point of view, the strict proof is not given here. Instead, we evaluate the validity of the solution method mainly by comparing the solutions obtained in this paper with those of other literature. The solutions obtained in Chapter 3 are examined as follows.

(1) As stated in Sections of 3.1, 3.6 and 3.7, the solutions obtained by this method are the same as those in other literature using the method of Laplace transformation. 
(2) In Sec. 3.2, the solution obtained by this method is the same as that obtained by a simple calculation using the result of Sec. 3.1 .

(3) In Sec. 3.3, the solution obtained by this method is the same as that obtained by a simple calculation based on the solutions of Sec. 3.1 and Sec. 3.2.

(4) In Sec. 3.4, the solution is obtained based on the solution of Sec. 3.3, and is the same as that by other literature using the method of Laplace transformation.

(5) In Sec. 3.5, the solution is obtained by a simple calculation based on the result of Sec. 3.3.

Based on the above considerations, we have the following conclusion:

As for the items of (1) and (4), the formulas of the typical solutions by this method are the same as those of the solutions obtained by other literature using the method of Laplace transformation, which strongly supports the validity of the new solution method proposed in this paper. Item (5) also supports the validity of the new solution method.

\section{Usefulness of the method}

The proposed analytical method gives a new and simple tool for solving the problem of one-dimensional transient heat conduction in a slab of finite thickness. Examples of the usefulness of the proposed new method in the field of mechanical engineering are as follows:

(1) The method gives a simple and easy tool to construct the solution.

The conventional analytical method such as Laplace transformation for the solution is mathematically rather difficult and complicated to obtain the solution; construction of the solution would be not so easy. On the other hand, the method proposed in this paper gives a simple and easy tool to obtain the series solution; we could construct the solution easily by drawing the curves for the solution graphically; the method is very convenient.

(2) The method makes it easy to understand the solution.

By this method, we can easily draw the curves for the solution, from which we could deeply understand the characteristics of the series solution (e. g.: understanding of the convergence characteristics of the series solution).

(3) The method can be applied to other problems such as those with surface convection boundary condition, time dependent boundary condition, and initial temperature condition which depends on the location.

It is expected that with some modifications, the proposed method can also be applied to the problems with the above conditions as well as those presented in this paper.

(4) An exact solution for the problem in a finite slab with surface recession may be constructed.

Solving the problem of temperature distribution in a solid with surface recession is important in the design of a thermal protection material (ablator) of an atmospheric re-entry capsule. An analytical solution is available for the transient response of a semi-infinite solid initially at uniform temperature exposed to a step in surface temperature and to a step in surface recession rate (Baer and Ambrosio, 1961). Quasi-steady form for the solution is also available (Baer and Ambrosio, 1961) (Moyer and Rindal, 1967). The exact solution for a finite slab with the corresponding conditions is important. When we apply the proposed solution method to the problem of the semi-infinite solid with surface recession and modify the treatment of boundary conditions, there is a possibility that the corresponding exact solution for a finite slab which has not ever been known can be constructed.

(5) An approximate solution for the problem in a finite slab with the boundary condition according to the fourth-power radiation law may be constructed.

The approximate solution in a semi-infinite solid with the boundary condition according to the fourth-power radiation law is given by the integral method (Ozisik, 1989). When we apply the proposed solution method to the above problem and modify the treatment of boundary conditions, there is a possibility that the corresponding approximate solution for a finite slab which has not ever been known can be constructed.

\section{Conclusions}

A new simple analytical method for solving the problem of one-dimensional transient heat conduction in a slab of finite thickness is proposed, in which the initial temperature $T_{\mathrm{i}}$ is assumed zero or constant and the boundary surfaces are assumed to be at constant temperature, constant heat flux, or insulated. When $T_{\mathrm{i}}$ is not zero, the problem is 
transformed to the case of $T_{\mathrm{i}}=0$. Thermo-physical properties of the materials are assumed constant.

In this method, the solution is expressed by an infinite series representation, where each term is the temperature function (the temperature solution) of the corresponding initial-boundary value problem for the semi-infinite solid. Each semi-infinite solid extends to infinity in the positive or negative direction of the $x$ axis and the surface is located at various positions along the $x$ axis. Each term and the partial sum in the infinite series automatically satisfy the heat conduction equation and the initial condition. The solution is constructed so that the boundary values of the partial sum converge to those of the boundary conditions as the number of terms increases to infinity. The construction of the solution is easy to conduct if we draw the graphic curves of the temperature functions for several terms and the sum of them.

The solution method was applied to various one-dimensional initial-boundary value problems. The formulas of the typical solutions by this method were found to be the same as those of the solutions obtained by other literature using the method of Laplace transformation, which supports the validity of the new solution method proposed in this paper.

For an initial-boundary value problem of a slab, if only the temperature functions for the corresponding semi-infinite solids are known, we could construct the solution for the slab easily by using this method. The solution method makes it easy to understand the characteristics of the solution.

It seems that with some modification, this method could also be applied to more complicated problems (e. g.: surface convection boundary condition, time dependent boundary condition, initial temperature condition which depends on the location, boundary condition with surface recession and fourth power radiation boundary condition). There is a possibility that a new series solution which has not ever been known may be constructed by using this method.

\section{References}

Baer, D and Ambrosio, A., Heat conduction in a semi-infinite slab with sublimation at the surface, Planetary and Space Science, Vol.4, (1961), pp. 436-446.

Brown, J. W. and Chuchill, R. V., Fourier Series and Boundary Value Problems (1993), pp. 110-117, 129-140, 193-195 and 234-238, 5th ed., McGraw-Hill International Editions, Mathematics and Statistics Series, McGraw-Hill.

Chapra, S. C. and Canale, R. P., Numerical Methods for Engineers (2010), pp. 871-886, 6th ed., McGraw-Hill.

Carslaw, H. S. and Jaeger, J. C., Conduction of Heat in Solid (1959), pp. 60, 75, 112-113 and 308-310, 2nd ed. Oxford University Press.

Churchill, R. V., Operational Mathematics (1958), pp. 128-143, 2nd ed. International Student Edition, McGraw-Hill, Kogakusha.

Constanda, C., Solution Techniques for Elementary Partial Differential Equations (2002), Chapters 4-10, Chapman \& Hall/CRC.

Farlow, S. J., Partial Differential Equations for Scientists and Engineers (1993), pp. 1-111 and 309-330, Dover.

Incropera, F. P., Dewitt, D. P., Bergman, T. L. and Lavine, A. S., Fundamentals of Heat and Mass Transfer (2007), pp. 283-287, Sixth Ed. John Wiley \& Sons.

Lewis, R. W., Nithiarasu, P. and Seetharamu, K. N., Fundamentals of the Finite Element Method for Heat and Fluid Flow (2004), pp. 152-162, John Wiley \& Sons.

Moyer, C.B, and Rindal, R. A., An analysis of coupled chemically reacting boundary layer and charring ablator, Part II , finite difference solution for the in-depth response of charring materials considering surface chemical and energy balances, NASA CR-1061, (1967), pp. B-7.

Ozisik, M. N., Boundary Value Problems of Heat Conduction (1989), pp. 318-323, Dover. 